\title{
Soliton appearing in boson-fermion mixture at the third order of the interaction radius
}

\author{
K. V. Zezyulin. . P. A. Andreev $]^{\dagger}$ and L. S. Kuz'menkov \\ Physics Faculty, Moscow State University, Moscow, Russian Federation.
}

\begin{abstract}
In this paper we consider an ultra-cold mixture of boson and fermion atoms on the basis of quantum hydrodynamics. Small perturbations in such systems are being analyzed. A possibility is shown for soliton solutions of a new type to appear if the third order of the interaction radius is taken into account in the analysis of interactions. A fermion-fermion interaction occurs in explicit form if this approximation is accepted. The conditions that lead to occurrence of this type of soliton in a mixture of boson and fermion atoms were investigated. Restrictions on the fermion-fermion interaction were found that are necessary for this kind of perturbations to appear in the system. Conditions determining whether perturbances would be a condensed soliton or a rarefied soliton are shown. Requirements to the experimental detection of a new soliton type in boson-fermion mixture are considered.
\end{abstract}

\section{INTRODUCTION}

Experimental and theoretical approaches to the physics of quantum gases have been continued to grow in recent times, and various nonlinear structures has been studied there [1]- [7]. Methods for producing Bose-Einstein condensate and investigating its properties have been developed [8]. This showed stable conditions to exist both for repulsion and attraction between the atoms, for example, in a Bose condensate of ${ }^{7} \mathrm{Li}$ atoms [9]. A mixture of Bose and Fermi degenerate gases, where interaction between atoms may be tuned from repulsion to attraction, is considered in [10]. Papers $[10,11]$ have been devoted to investigations of ultra-cold boson-fermion mixtures. A branch of physics that works on producing of a stable boson-fermion mixture is being developed as well [12].

A lot of interest has been attracted in recent years to the investigation of soliton solutions. Papers devoted to properties of different types of solitons are constantly being emerged. As to the present time a bright soliton has been produced in a Bose-Einstein condensate with a repulsive interaction between atoms. A soliton of this type, has been produced, for example, in the condensate of ${ }^{87} R b$ atoms in a weak periodic potential [13]. The formation of a bright soliton in the condensate of ${ }^{7} \mathrm{Li}$ atoms is considered in [9, 14] for a quasi-1D case. Properties of a dark soliton have been studied in [15]. They show experimental generation of a dark soliton in a cigar-shaped condensate of ${ }^{87} R b$ atoms with the use of phase imprinting technique. The study of a dark soliton in Bose-Einstein condensate inside a vortex ring is described in [16]. In addition to one-dimension solitonic perturbations in a Bose-Einstein condensate, three-dimension perturbations are also attracting attention [17]. A possibility was shown for solitonic perturbations to exist in a Bose-Einstein condensate with dipole-dipole interaction [18]. In recent years predictions have been made that solitons of bright-bright, dark-bright and dark-dark types may exist in a two-component BEC [19]. Solitons in boson-fermion mixture is special and interesting item. Perturbations in boson-fermion mixtures have also been studied and the existence of a bright soli- ton in boson-fermion mixture has been proved in [10, 20].

In the present work we study ultra-cold boson-fermion mixtures. Examples of such mixtures are ${ }^{7} \mathrm{Li}-{ }^{6} \mathrm{Li}$ [21, 22], ${ }^{23} \mathrm{Na}-{ }^{6} \mathrm{Li}$ [23] and ${ }^{87} \mathrm{Rb}-{ }^{40} \mathrm{~K}$ [24]. The possibility of new solitonic perturbations in a mixture of boson and fermion atoms with short-range interaction potentials is analyzed in particular. Theoretical analysis at higher order of interaction account accuracy allows prediction of new types of soliton solutions [25]. This approximation can be derived with the aid of quantum hydrodynamics approach, which has been developed in recent decade [25-27]. The set of quantum hydrodynamic equations describing an ultra-cold mixture of boson and fermion atoms may be derived from a many-particle Schrodinger equation [25]. In [28], for example, they used this approach to study how the shape of a bright soliton changes as interactions in a Bose-Einstein condensate are considered more precisely. The method of quantum hydrodynamics is very useful in different areas of physics. Namely, it was quantum plasma [26], [29], plasma of particles with the own magnetic moment [30]- [34], relativistic quantum plasma [35], ultracold Bose and Fermi gases with nonlocal interaction [25], quantum particles with electrical [36], [27] and magnetical [37] polarization, particularly being in the BEC state [27], [37], the graphene electrons [38] and $\mathrm{BEC}$ of graphene excitons [39].

Most of theoretical papers devoted to the ultracold fermions contain nonlinear Schrodinger equation (an ana$\log$ of Gross-Pitaevskii equation using for bosons) which does not include interaction [40]- [49]. It is connected with the fact that the first Born approximation gives no contribution in interaction of ultracold fermions. This equation undoubtedly accounts the Fermi pressure contribution caused by Pauli principle. It is very interesting to understand a role of interaction in fermion systems. For example, vortices in a strongly interacting gas of fermionic atoms on the BEC- and the BCS-side of the Feshbach resonance was experimentally studied in Ref. [50]. Presented in our paper model based on the quantum hydrodynamics method contain fermion-fermion interaction whose form was derived directly from the Schrodinger equation [25]. 
In this paper we discuss the existence of new types of solitons in a mixture of boson and fermion atoms and determine the conditions of their experimental detection. A substantial role in our analysis is given to the fermion-fermion interaction coefficient, which appears in the analysis of the third order of the interaction range. A perturbation method introduced by Washimi [51], [52] is used here to find solitonic perturbations. An analogous method was used for a spinor-1 BEC studying [53]. Other methods for weaknonlinear analysis of BEC have been considered in literature. For example, in Ref. [54] the Krylow-BogoliubovMitropolskii method was used for nonlinear frequency shift calculation. It was shown that account of interaction up to TOIR approximation leads to the new solitons in BEC [55].

Our paper is organized as follows. In Sect. 2 we present basic equation and describe using model. In Sect. 3 we study solitons in boson-fermion mixture which appearing due to interaction account up to TOIR approximation. In Sect. 3 detailed analysis of conditions of a "fermion" soliton existence is presented. In Sect. 4 condition of a "boson" soliton existence is described. In Sect. 5 brief summary of obtained results is presented.

\section{THE MODEL}

Let's consider a mixture of ultra-cold boson and fermion atoms having our focus on one-dimension perturbations. The set of quantum hydrodynamic equations consists of the following equations: the continuity equation for bosons

$$
\frac{\partial n_{b}}{\partial t}+\frac{\partial\left(n_{b} v_{b}\right)}{\partial x}=0
$$

the momentum balance equation for bosons

$$
\begin{aligned}
m_{b} n_{b} \frac{\partial v_{b}}{\partial t}+ & \frac{1}{2} m_{b} n_{b} \frac{\partial v_{b}^{2}}{\partial x}-\frac{\hbar^{2}}{2 m_{b}} n_{b} \frac{\partial}{\partial x}\left(\frac{1}{\sqrt{n_{b}}} \frac{\partial^{2} \sqrt{n_{b}}}{\partial x^{2}}\right) \\
& -\Upsilon_{b b} n_{b} \frac{\partial n_{b}}{\partial x}-\frac{1}{16} \Upsilon_{2 b b} \frac{\partial^{3} n_{b}^{2}}{\partial x^{3}} \\
& =\Upsilon_{b f} n_{b} \frac{\partial n_{f}}{\partial x}+\frac{1}{2} \Upsilon_{2 b f} n_{b} \frac{\partial^{3} n_{f}}{\partial x^{3}}
\end{aligned}
$$

the continuity equation for fermions

$$
\frac{\partial n_{f}}{\partial t}+\frac{\partial\left(n_{f} v_{f}\right)}{\partial x}=0
$$

and the momentum balance equation for fermions

$$
\begin{aligned}
m_{f} n_{f} \frac{\partial v_{f}}{\partial t} & +\frac{1}{2} m_{f} n_{f} \frac{\partial v_{f}^{2}}{\partial x}-\frac{\hbar^{2}}{2 m_{f}} n_{f} \frac{\partial}{\partial x}\left(\frac{1}{\sqrt{n_{f}}} \frac{\partial^{2} \sqrt{n_{f}}}{\partial x^{2}}\right) \\
+ & \frac{3}{8} \Upsilon_{2 f f} \frac{\partial n_{f}}{\partial x} \frac{\partial n_{f}^{2}}{\partial x}-\frac{3}{8} n_{f} \Upsilon_{2 f f} \frac{\partial^{3} n_{f}}{\partial x^{3}}
\end{aligned}
$$

$$
\begin{gathered}
+\frac{1}{2}\left(3 \pi^{2}\right)^{2 / 3} \Upsilon_{2 f f} \frac{\partial n_{f}^{8 / 3}}{\partial x}+\frac{\hbar^{2}}{5 m_{f}}\left(3 \pi^{2}\right)^{2 / 3} \frac{\partial n_{f}^{5 / 3}}{\partial x} \\
=\Upsilon_{b f} n_{f} \frac{\partial n_{b}}{\partial x}+\frac{1}{2} \Upsilon_{2 b f} n_{f} \frac{\partial^{3} n_{b}}{\partial x^{3}} .
\end{gathered}
$$

The equations given above utilize following designations: $m_{b}, m_{f}$ - masses of boson and fermion atoms, respectively; $n_{b}, n_{f}$ - concentrations of bosons and fermions; $v_{b}, v_{f}$ respective velocity fields. $\Upsilon_{b b}, \Upsilon_{b f}$ - coefficients of bosonboson and boson-fermion interactions at the first order of the interaction range. $\Upsilon_{2 b b}, \Upsilon_{2 b f}, \Upsilon_{2 f f}$ - coefficients of boson-boson, boson-fermion and fermion-fermion interactions at the third order of the interaction range. Interaction coefficients can be defined with following equations:

$$
\begin{aligned}
& \Upsilon_{i j}=\frac{4 \pi}{3} \int d r r^{3} \frac{\partial U_{i j}(r)}{\partial r} \\
& \Upsilon_{2 i j}=\frac{4 \pi}{15} \int d r r^{5} \frac{\partial U_{i j}(r)}{\partial r} .
\end{aligned}
$$

Presented here hydrodynamics equations correspond to the system of two nonlinear Schrodinger equations one for bosons another for fermions [25]. These nonlinear Schrodinger equations are nonlocal and integrodifferential. The nonlinear Schrodinger equation for Bose particles is a generalization of Gross-Pitaevskii equation. This generalization appear due to more detailed account of short-range interaction up to third order on interaction radius. For the first time a nonlocal Gross-Pitaevskii equation were derived in Ref.s. [56], [57]. This equation does not contain integral terms. Brief comparison for different nonlocal generalization of the Gross-Pitaevskii equation is discussed in ref. [28].

Coefficients $\Upsilon_{b b}$ and $\Upsilon_{b f}$ are related to interactions coefficients $g_{b b}$ and $g_{b f}$ of the Gross-Pitaevskii equation as follows:

$$
\Upsilon_{b b}=-g_{b b}, \Upsilon_{b f}=-g_{b f}
$$

It should be noted that the following relationship exists between interaction coefficients and scattering amplitude $a_{i j}$

$$
\Upsilon_{i j}=-\frac{4 \pi \hbar^{2} a_{i j}}{m}
$$

All equations above are approximated up to the third order of the interaction range. If terms containing $\Upsilon_{2 b b}$, $\Upsilon_{2 b f}, \Upsilon_{2 f f}$, are neglected then the resulting equation set corresponds to the first-order approximation (the GrossPitaevskii approximation) which has been used, for example, in [11]. It was shown in [25] that the approximation is possible that derives coefficients $\Upsilon_{2 b b}, \Upsilon_{2 b f}$ from $\Upsilon_{b b}$, $\Upsilon_{b f}$. The explicit form of this approximation is:

$$
\Upsilon_{2 b b} \simeq r_{0}^{-2} \Upsilon_{b b}
$$

and

$$
\Upsilon_{2 b f} \simeq r_{0}^{-2} \Upsilon_{b f},
$$

where $r_{0}$ - is a constant of the same order of magnitude as the atomic radius. 


\section{THE SOLITON SOLUTION IN A BOSON-FERMION MIXTURE.}

The equation set in question should be expected to have soliton solutions of a new type due to more accurate recognition of atomic interactions. The method of perturbations may be applied to find this soliton [51, 58]. According to this method all hydrodynamic values may be represented as:

$$
\begin{gathered}
n_{b}=n_{0 b}+\varepsilon n_{1 b}+\varepsilon^{2} n_{2 b}+\ldots, \\
n_{f}=n_{0 f}+\varepsilon n_{1 f}+\varepsilon^{2} n_{2 f}+\ldots, \\
v_{b}=\varepsilon v_{1 b}+\varepsilon^{2} v_{2 b}+\ldots, \\
v_{f}=\varepsilon v_{1 f}+\varepsilon^{2} v_{2 f}+\ldots
\end{gathered}
$$

We also performed the following "scaling" of variables:

$$
\xi=\varepsilon^{1 / 2}(x-U t)
$$

and

$$
\tau=\varepsilon^{3 / 2} U t
$$

The latter expression introduces so-called "slow" time.

The following expression of the phase velocity $U$ can be derived from equations (14) in the first order of the small parameter $\varepsilon$ :

$$
\begin{gathered}
U_{ \pm}^{2}=\frac{1}{2 m_{b} m_{f}}\left(\Theta m_{b} n_{0 f}-m_{f} n_{0 b} \Upsilon_{b b}\right) \\
\pm \frac{1}{2 m_{b} m_{f}}\left(\left(\Theta m_{b} n_{0 f}-m_{f} n_{0 b} \Upsilon_{b b}\right)^{2}\right. \\
\left.\quad+4 m_{b} m_{f} n_{0 b} n_{0 f}\left(\Theta \Upsilon_{b b}+\Upsilon_{b f}^{2}\right)\right)^{1 / 2}
\end{gathered}
$$

where

$$
\Theta \equiv \frac{\left(3 \pi^{2}\right)^{2 / 3}}{n_{0 f}}\left(\frac{\hbar^{2}}{3 m_{f}} n_{0 f}^{2 / 3}+\frac{4}{3} \Upsilon_{2 f f} n_{0 f}^{5 / 3}\right) .
$$

Quantity $\Theta$ describes the contribution of fermion dynamics in boson-fermion mixture. $\Theta$ consists of two parts. The first one appears from Fermi pressure and the second term presents the fermion-fermion short-range interaction which arises at interaction account up to the third order of the interaction radius. In formula (15) the quantity under radical is positive.

Since our mixture is comprised by two interacting subsystems, boson atoms and fermion atoms, two different values of phase velocity are obtained here. We call phase velocity $U_{+}$which has "+" sign before the radical, the "fermion branch" of the solution. Phase velocity $U_{-}$is called the "boson branch" of the solution by analogy. We introduced these terms to emphasize the fact that in the absence of boson-fermion interactions $\Upsilon_{b f}=0$ the system can be divided in two subsystems of bosons and fermions, respectively, which do not interact. Phase velocity $U_{+}$corresponds in this case to fermions

$$
U_{+}^{2}=\frac{n_{0 f}}{m_{f}} \Theta
$$

and $U_{-}$corresponds to bosons

$$
U_{-}^{2}=-\frac{n_{0 b}}{m_{b}} \Upsilon_{b b}
$$

We can see that $U_{-}^{2}>0$ for particles with repulsive interaction $\Upsilon_{b b}<0$.

In the second order of the small parameter $\varepsilon$ we derive the Korteweg - de Vries equation for small perturbations of boson concentration in a boson-fermion mixture:

$$
p \frac{\partial n_{1 b}}{\partial \tau}+q \frac{\partial^{3} n_{1 b}}{\partial \xi^{3}}+s n_{1 b} \frac{\partial n_{1 b}}{\partial \xi}=0,
$$

where coefficient $p$ of the term containing slow time

$$
p=2 U^{2}\left(\Theta m_{b} n_{0 f}-\Upsilon_{b b} n_{0 b} m_{f}-2 U^{2} m_{b} m_{f}\right),
$$

coefficient $q$ of the term that corresponds to dispersion

$$
\begin{gathered}
q=-\left(\Theta n_{0 f}-m_{f} U^{2}\right) \times \\
\times\left(\frac{\hbar^{2}}{4 m_{f}}+\frac{1}{8} \Upsilon_{2 b b} n_{0 b}-\frac{1}{2} \Upsilon_{2 b f} n_{0 b} \frac{\Upsilon_{b b}}{\Upsilon_{b f}}-\frac{1}{2} \Upsilon_{2 b f} \frac{U^{2} m_{b}}{\Upsilon_{b f}}\right) \\
+\left(\frac{\hbar^{2}}{4 m_{f}}+\frac{1}{4} \Upsilon_{2 f f} n_{0 f}\right)\left(n_{0 b} \Upsilon_{b b}+U^{2} m_{b}\right) \\
+\frac{1}{2} \Upsilon_{2 b f} \Upsilon_{b f} n_{0 b} n_{0 f}
\end{gathered}
$$

and coefficient $s$ of the non-linear term

$$
\begin{gathered}
s=3 \frac{U^{2} m_{b}}{n_{0 b}}\left(\Theta n_{0 f}-U^{2} m_{f}\right)+\Upsilon_{b f}\left(\Upsilon_{b b} n_{0 b}+U^{2} m_{b}\right) \\
+\left(\Upsilon_{b b}+\frac{U^{2} m_{b}}{n_{0 b}}\right)^{2} \frac{n_{0 b}}{\Upsilon_{b b}} \times \\
\times\left(2 \frac{U^{2} m_{f}}{n_{0 f}}+\frac{20}{9}\left(3 \pi^{2} n_{0 f}\right)^{2 / 3} \Upsilon_{2 f f}+\frac{\hbar^{2}}{9 m_{f}} \frac{\left(3 \pi^{2}\right)^{2 / 3}}{n_{0 f}^{1 / 3}}\right) .
\end{gathered}
$$

The soliton solution of the Korteweg - de Vries equation (17) is well known in the form of

$$
n_{1 b}=\frac{3 p V}{s} \cdot \frac{1}{\cosh ^{2}\left(\sqrt{\frac{V p}{4 q}} \eta\right)},
$$

where $\eta=\xi-V \tau$.

Below we discuss perturbations in bosons implying that perturbations in fermions are similar to them due to the following linear relationship between $n_{1 b}$ and $n_{1 f}$ :

$$
\Upsilon_{b f} n_{1 f}=-\left(\Upsilon_{b b}+\frac{U^{2} m_{b}}{n_{0 b}}\right) n_{1 b}
$$

Soliton pairs (in bosons and fermions, respectively) of two types may evolve: dark-bright and dark-dark. 


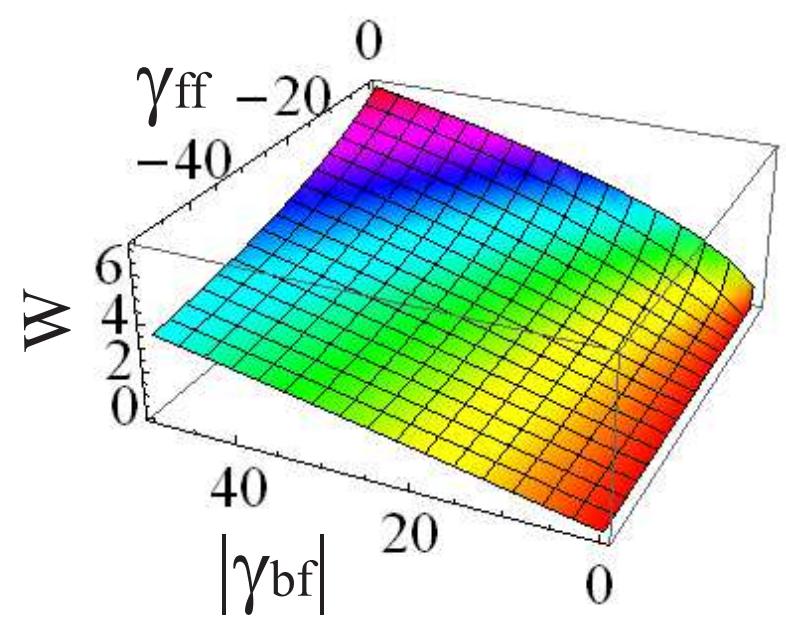

FIG. 1. (Color online) The relationship between dimensionless phase velocity $W_{f}$ and coefficients of fermion-fermion interaction $\gamma_{b f}$ and boson-fermion interaction $\gamma_{b f}$ for fermion branch. Figure is made for the following parameters of the system: $\gamma_{b b}=$ $-10^{-2}, \mu=1, N=1, B_{b f}=10^{-3}$, and $B_{b b}=-10^{-4}$. This plot and plots 2-4 made for the repulsion between Bose particles $\gamma_{b b}<0$. Dimensionless phase velocity $W_{f}$ has analogous form in the case of attraction between Bose particles.

\section{THE FERMION BRANCH OF THE SOLUTION.}

Let's investigate "fermion branch" of the solution, where phase velocity $U$ from the equation (15) is taken with " + " sign.

If boson-fermion interactions are "strong" enough compared to boson-boson interactions, then $U^{2}>0$. We use apply assumption to the fermion branch below.

For numerical analysis of our results we introduce following dimensionless variables: dimensionless phase velocity $W_{+}=m_{f} U_{+} /\left(\hbar n_{0 f}^{1 / 3}\right)$, mass rate $\mu=m_{f} / m_{b}$, concentrations rate $N=\sqrt{n_{0 b} / n_{0 f}}$, and dimensionless interaction constants $\gamma_{b b}=m_{f} n_{0 b} \Upsilon_{b b} /\left(n_{0 f}^{1 / 3} \hbar\right)^{2}, \gamma_{b f}=$ $m_{f} \sqrt{n_{0 b} n_{0 f}} \Upsilon_{b f} /\left(n_{0 f}^{1 / 3} \hbar\right)^{2}, \quad \gamma_{f f}=m_{f} n_{0 f} \Upsilon_{2 f f} / \hbar^{2}$, $B_{b b}=m_{f} n_{0 b} \Upsilon_{2 b b} / \hbar^{2}$, and $B_{b f}=n_{o f}^{2 / 3} \Upsilon_{2 b f} / \Upsilon_{b f}$.

Phase velocity for fermion branch is shown at Fig. 1.

It can be shown that

$$
p<0
$$

at any allowable values of physical parameters. It follows from numerical analysis of $p$.

As a starting point we considered the first order of the interaction range, neglecting the contribution of terms that are proportional to coefficients $\Upsilon_{2 b b}, \Upsilon_{2 b f}, \Upsilon_{2 f f}$ in equations (2) and (4).

In this case the coefficient $q$ takes the following value:

$$
q=\alpha \frac{\hbar^{2}}{4 m_{b}}+\beta \frac{\hbar^{2}}{4 m_{f}}>0,
$$

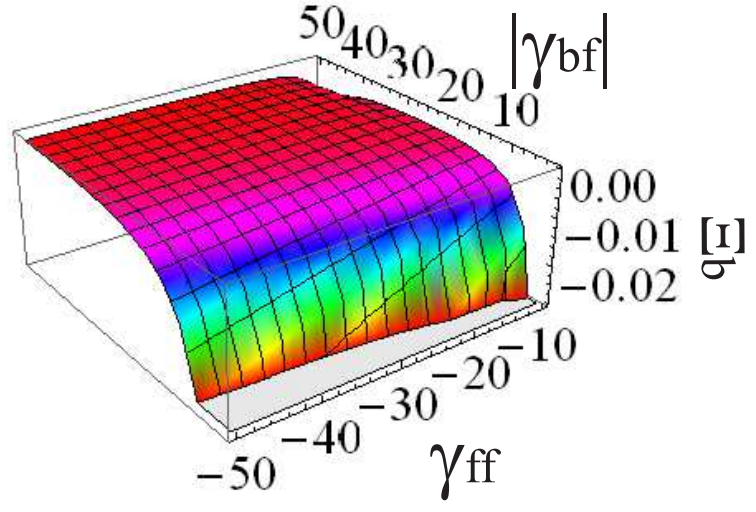

FIG. 2. (Color online) The relationship between soliton amplitude $\Xi_{b}$ of Bose subsystem and the coefficient of boson-fermion interaction $\gamma_{b f}$ and fermion-fermion interaction $\gamma_{f f}$ for fermion branch. Figure is made for the following parameters of the system: $\gamma_{b b}=-10^{-2}, \mu=1, N=1, B_{b f}=10^{-3}$, and $B_{b b}=-10^{-4}$.

where $\alpha>0$ and $\beta>0$. Evident form for $\alpha$ and $\beta$ to be

$$
\alpha=\frac{1}{2 m_{b}}\left(-\left(\Theta m_{b} n_{0 f}+\Upsilon_{b b} m_{f} n_{0 b}\right)\right.
$$

$\left.+\sqrt{\left(\Theta m_{b} n_{0 f}+\Upsilon_{b b} m_{f} n_{0 b}\right)^{2}+4 m_{b} m_{f} n_{0 b} n_{0 f}\left(\Upsilon_{b f}\right)^{2}}\right)$,

and

$$
\begin{gathered}
\beta=\frac{1}{2 m_{f}}\left(\left(\Theta m_{b} n_{0 f}+\Upsilon_{b b} m_{f} n_{0 b}\right)\right. \\
\left.+\sqrt{\left(\Theta m_{b} n_{0 f}+\Upsilon_{b b} m_{f} n_{0 b}\right)^{2}+4 m_{b} m_{f} n_{0 b} n_{0 f}\left(\Upsilon_{b f}\right)^{2}}\right) .
\end{gathered}
$$

Expressions (21), (23) and (24) make it clear that no soliton exists in the boson-fermion mixture in the GrossPitaevskii approximation, as the radicand in (21) is negative.

So, we took into account the third order by the interaction radius.

Now the coefficient $q$ has the following form:

$$
q=\beta\left(\frac{\alpha}{\beta} \frac{\hbar^{2}}{4 m_{b}}+\frac{\hbar^{2}}{4 m_{f}}+\frac{1}{4} n_{0 f} Y_{2 f f}\right)+\ldots
$$

It means that if the repulsion of fermions is strong enough (i.e. the coefficient of fermion-fermion interaction $Y_{2 f f}<$ 0 ) the $q$ value would be negative. Strong fermion-fermion interactions in the boson-fermion mixture make possible perturbations of a new type, which do not occur in the first order of the interaction range.

In the case the considered perturbations of concentration are small, solitons of two kinds may arise: solitons of fermion rarefication and of fermion compression. The 


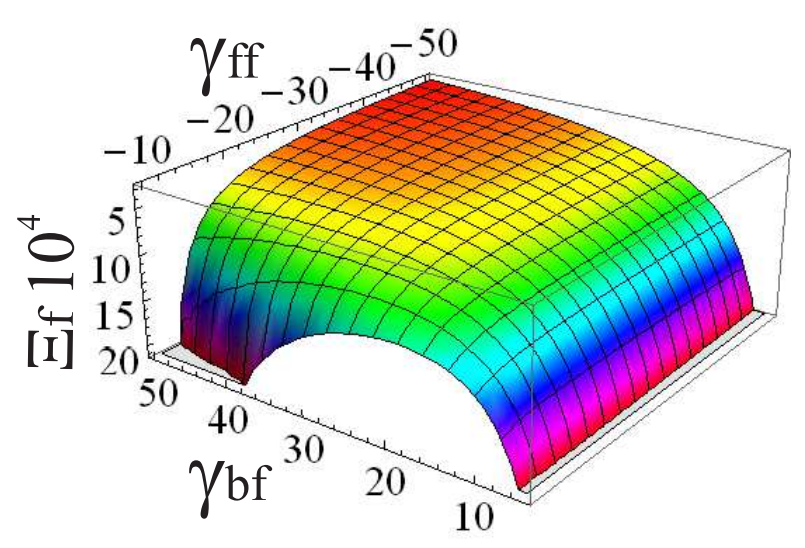

FIG. 3. (Color online) The dependence curve of the soliton amplitude $\Xi_{f}$ of Fermi subsystem vs the coefficient of fermionfermion interaction $\gamma_{f f}$ and boson-fermion interaction $\gamma_{b f}$ for the fermion branch. The plot is built with following parameters: $\gamma_{b b}=-10^{-2}, \mu=1, N=1, B_{b f}=10^{-3}$, and $B_{b b}=-10^{-4}$.

soliton type is determined by the sign of its dimensionless amplitude of bosons

$$
\Xi_{b}=\frac{3 p V}{s n_{0 b}}
$$

and fermions $\Xi_{f}$ which connected with the $\Xi_{b}$ by formula (22).

The dependence between amplitude of soliton in the boson subsystem and the coefficient of boson-fermion interaction $\Upsilon_{b f}$ is shown at Fig.s 2-4. If the coefficient value is high positive (it corresponds to a strong attraction between boson and fermion atoms) then boson perturbations would be solitons of depression (dark soliton, it is shown at Fig.2) and, as it follows from (22), fermion perturbations would be solitons of compression (bright soliton) for $\gamma_{b f}>0$ or soliton of depression (dark soliton) for $\gamma_{b f}<0$ (it is shown at Fig.s 3 and 4). These are dark-bright and dark-dark solitons in a two-component system of bosons and fermions. So, a strong repulsion between bosons and fermions (i.e. negative values of the $\Upsilon_{b f}$ coefficient) would lead to solitons of compression of bosons and fermions, which correspond to a dark-dark soliton.

The dimensionless soliton width $D$ can be defined by the following expression:

$$
D=\sqrt{\frac{4 q n_{0 f}^{2 / 3}}{V p}}
$$

It follows from (18) and (27) that soliton width depends on the fermion-fermion interaction. This dependence is plotted at Fig. 5. Note that the surface's shape does not change along with the sign of $\gamma_{b f}$ as the expression for the soliton width $D$ contains the coefficient of boson-fermion interaction in a squared form.

There is interesting behavior of this solution for attractive boson-boson interaction. In this case soliton exist

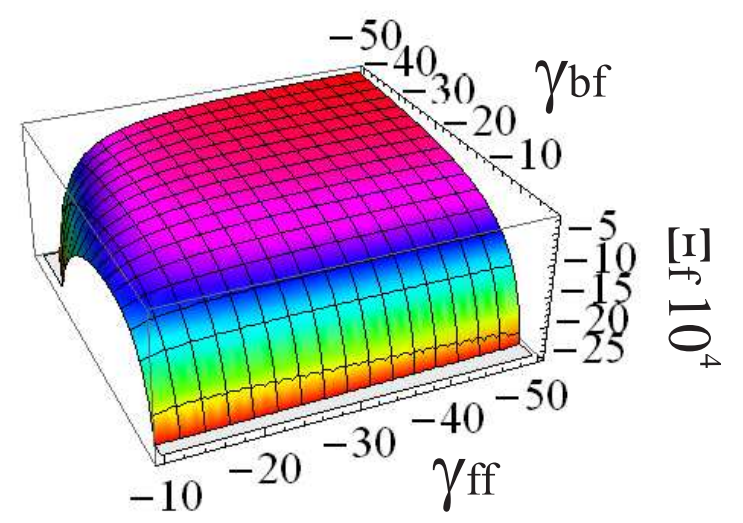

FIG. 4. (Color online) The dependence curve of the soliton amplitude $\Xi_{f}$ of Fermi subsystem vs the coefficient of fermionfermion interaction $\gamma_{f f}$ and boson-fermion interaction $\gamma_{b f}$ for the fermion branch in the case negative $\gamma_{b f}$ that corresponds to repulsion between Bose and Fermi particle. The plot is built with following parameters: $\gamma_{b b}=-10^{-2}, \mu=1, N=1$, $B_{b f}=10^{-3}$, and $B_{b b}=-10^{-4}$.

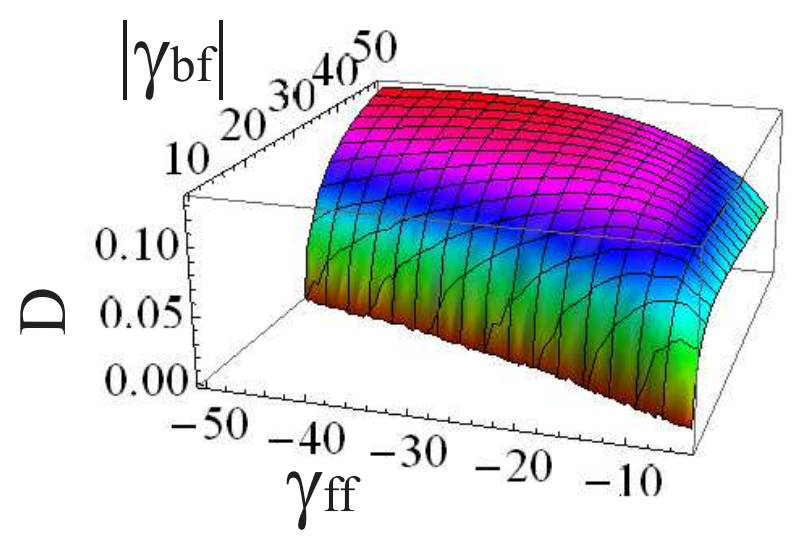

FIG. 5. (Color online) The dependence curve of the soliton width $D$ vs the coefficient of fermion-fermion interactions $\gamma_{f f}$ and the coefficient of boson-fermion interaction $\gamma_{b f}$ for the fermion branch expressed in reduced values. The plot is built with following parameters: $\gamma_{b b}=-10^{-2}, \mu=1, N=1, B_{b f}=10^{-3}$, and $B_{b b}=-10^{-4}$. Analogous dependence take place at $\gamma_{b b}>0$ for $\gamma_{b b} \sim 10^{-2} \div 10^{-3}$ in the area of large boson fermion interaction $\left|\gamma_{b f}\right|>10$

in two areas of system parameters. For the large $\left|\gamma_{b f}\right|$ $\left(\left|\gamma_{b f}\right|>10\right)$, function $D\left(\gamma_{f f}, \gamma_{b f}\right)$ is analogous to the same presented at Fig. 5. But for $\gamma_{b b}>0$ there is an area at the small $\left|\gamma_{b f}\right|$ where exist soliton solution. In this area soliton width is large, much more than 1 . It is presented at Fig.s 6-8.

In the case $\gamma_{b b}>0$ and $\left|\gamma_{b f}\right|<1$ amplitude of bosons is positive $\Xi_{b}>0$. So, we have bright soliton in subsystem of bosons Fig. 6. From Fig. 7 we can see that if $\gamma_{b f}<0$ amplitude of soliton in Fermi subsystem is positive. Thus, we have found bright soliton in Fermi subsystem. If $\gamma_{b f}>$ 


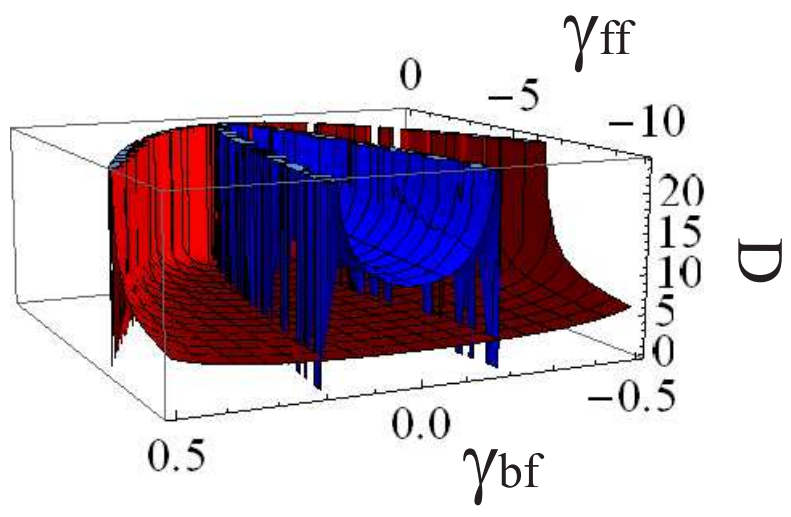

FIG. 6. (Color online) The dependence curve of the soliton width $D$ the coefficient of boson-fermion $\gamma_{b f}$ and fermion-fermion $\gamma_{f f}$ interaction for the fermion branch. This plot made for the attraction between Bose particles $\gamma>0$. The plot is built for two values of $\gamma_{b b}$ (for blue-narrow surface we took $\gamma_{b b}=10^{-3}$, and for redwide surface we used $\gamma_{b b}=10^{-2}$ ) with following parameters: $\mu=1, N=1, B_{b f}=10^{-4}$, and $B_{b b}=-10^{-4}$.

0 we have dark soliton in Fermi subsystem. In the result in boson-fermion mixture we have bright-dark soliton for $\gamma_{b f}<0$, and bright-bright soliton for $\gamma_{b f}>0$.

Considered in the paper values of interaction parameters may be obtained using Feshbach resonance [59, 60]. It follows from (5) and (6), that changing potential of atomic interaction changes both $\Upsilon_{i j}$ and $\Upsilon_{2 i j}$ coefficients. Note that soliton of this type occurs due to boson-fermion interactions.

\section{THE BOSON BRANCH OF THE SOLUTION.}

To obtain the boson branch phase velocity $U$ from the equation (15) should be taken with "-" sign.

Using the first order of the interaction radius does not allow to predict solitonic perturbations in boson-fermion mixtures. It can be done only when the third order is taken into account. If the mixture of bosons and fermions divides into two subsystems that do not interact, bosons "do not feel" the presence of fermions, $\Upsilon_{b f}=0$, and the coefficient $q \simeq-\hbar^{2} / 4 m_{b}-n_{0 b} \Upsilon_{2 b b} / 8$ [55]. This means that repulsion of bosons $\left(\Upsilon_{2 b b}<0\right)$ must be strong enough to cause soliton formation in a subsystem of boson atoms.

If the interaction of bosons and fermions occurs, then the acceptable range of physical values, where the soliton formation is possible, changes. In boson-fermion mixture a soliton corresponding to boson branch exists at more large strength of boson-boson interaction, in comparison with the case when $\Upsilon_{b f}=0$. We have bright soliton solution $\left(\Xi_{b}>0\right)$ in Bose subsystem. In Fermi subsystem we find dark $\Xi_{f}<0$ (bright $\Xi_{f}>0$ ) soliton for repulsion (attraction) between Bose and Fermi particles

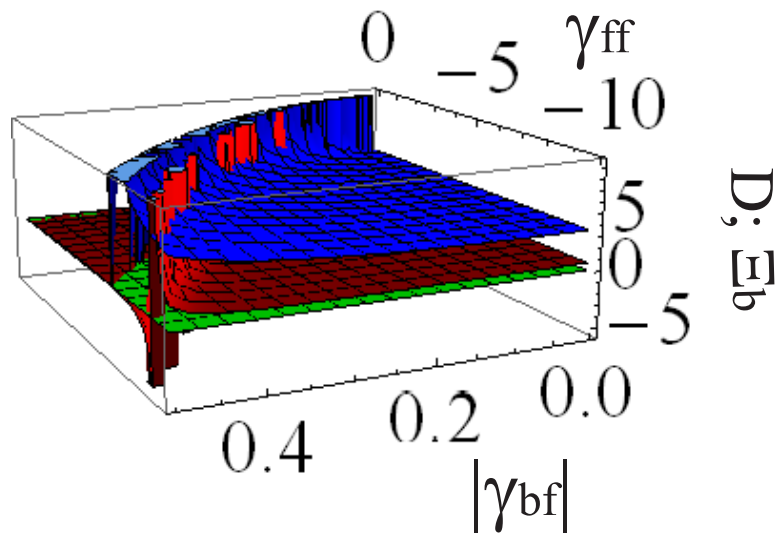

FIG. 7. (Color online) The dependence curve of the soliton width $D$ (blue-top surface) and amplitude for bosons $\Xi_{b}$ (red-middle surface) on the coefficient of boson-fermion $\gamma_{b f}$ and fermionfermion $\gamma_{f f}$ interaction for the fermion branch. Soliton exist if its width $D$ is positive. We present soliton width $D$ and amplitude for bosons $\Xi_{b}$ on the same plot to show the value of amplitude in the area where soliton exist. Green plane-lower surface present zero level. It show us that amplitude is positive. The plot is built with following parameters: $\gamma_{b b}=10^{-2}, \mu=1, N=1$, $B_{b f}=10^{-4}$, and $B_{b b}=-10^{-4}$.

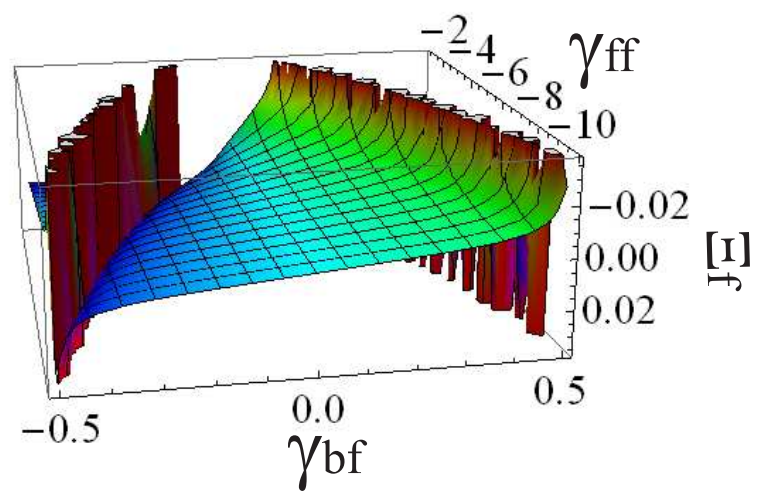

FIG. 8. (Color online) The dependence curve of the soliton width $D$ the coefficient of boson-fermion $\gamma_{b f}$ and fermion-fermion $\gamma_{f f}$ interaction for the fermion branch. The plot is built with following parameters: $\gamma_{b b}=10^{-2}, \mu=1, N=1, B_{b f}=10^{-4}$, and $B_{b b}=-10^{-4}$.

$\Upsilon_{b f}<0\left(\Upsilon_{b f}>0\right)$. We have got it because quantity $-\left(\Upsilon_{b b}+m_{b} U_{-}^{2} / n_{0 b}\right)$ presented by formula (22) is positive. Consequently, $\Xi_{f}$ has the same sign as $\Upsilon_{b f}$. So, in a mixture of bosons and fermions this type of soliton solution may exist.

Finally, we can note that two soliton solutions exists in boson-fermion mixture due to account of short range interaction up to the third order of the interaction radius. 


\section{CONCLUSION}

The quantum hydrodynamics approach is well suitable to derive equations that describe multiparticle quantum systems. In our case we applied the QHD approach to the ultra-cold mixture of boson and fermion atoms with shortrange interaction potential in order to analyze the possibility of small-scale solitonic perturbations. We show that solitons of two novel types may occur, which are related to fermion and boson branches of the solution, respectively.

In the fermion branch the soliton of a new type occurs due to boson-fermion interactions at the first order of the interaction range. Fermion-fermion interactions also play substantial role in formation of these perturbations. The type of soliton formed in the system (compressed or rarefied) is determined by the sign of boson-fermion interaction, i.e. repulsion or attraction, respectively. The dependence of fermion concentration on system characteristics was successfully derived. Consideration of the third order of the interaction range is the key factor that allowed prediction of a novel type of soliton solution. It also helped us to find new acceptable ranges of physical values, where the existence of soliton is possible for the boson branch of the solution in the boson-fermion mixture, compared to the system comprised of bosons only.

* konstantin.zez@mail.ru

$\dagger$ andreevpa@physics.msu.ru

$\ddagger$ 1sk@phys.msu.ru

[1] N. G. Parker, D. A. Smith, Phys. Rev. A 85, 013604 (2012).

[2] T. P. Billam, S. L. Cornish, and S. A. Gardiner, Phys. Rev. A 83, 041602(R) (2011).

[3] J. Abdullaev, A. S. Desyatnikov and E. A. Ostrovskaya,J. Opt. 13, 064023 (2011).

[4] U. Al. Khawaja and H. T. C. Stoof, New Journal of Physics 13, 085003 (2011).

[5] W. B. Cardosoa, A. T. Avelara, D. Bazeiab, Phys. Lett. A 374, 2640 (2010).

[6] L. Wen, Y. Zhang and J. Feng, J. Phys. B: At. Mol. Opt. Phys. 43, 225302 (2010).

[7] G. Filatrella, B. A. Malomed, and L. Salasnich, Phys. Rev. A 79, 045602 (2009).

[8] J. Denschlag, and et al., Science 287, 97 (2000).

[9] K. E. Strecker, G. B. Partidge, A. G. Truscott, R. G. Hulet, Nature 417, 9 (2002).

[10] T. Karpiuk, et al., Phys. Rev. Lett. 93, 100401 (2004).

[11] B. Ramachandhran, S. G. Bhongale, and H. Pu, Phys. Rev. A 83, 033607 (2011).

[12] Zeng-Qiang Yu, Shizhong Zhang, and Hui Zhai, Phys. Rev. A 83, 041603 (2011).

[13] B. Eiermann, Th. Anker, M. Albiez, M. Taglieber, P. Treutlein, K.-P. Marzlin, and M. K. Oberthaler, Phys. Rev. Lett. 92, 230401 (2004).

[14] L. Khaykovich, F. Schreck, G. Ferrari, T. Bourdel, J. Cubizolles, L. D. Carr, Y. Castin, C. Salomon, Science 296, 1290
(2002).

[15] S. Burger, K. Bongs, S. Dettmer, W. Ertmer, K. Sengstock, A. Sanpera, G. V. Shlyapnikov, and M. Lewenstein, Phys. Rev. Lett. 83, 5198 (1999).

[16] B. P. Anderson, et al., Phys. Rev. Lett. 86, 2926 (2001).

[17] A. M. Mateo, V. Delgado, B. A. Malomed, Phys. Rev. A 83, 053610 (2011).

[18] J. Cuevas, B. A. Malomed, P. G. Kevrekidis, and D. J. Frantzeskakis, Phys. Rev. A 79, 053608 (2009).

[19] G. Csire, D. Schumayer, and B. Apagyi, Phys. Rev. A 82, 063608 (2010).

[20] S. K. Adhikari and L. Salasnich, Phys. Rev. A 76, 023612 (2007).

[21] A. G. Truscott, K. E. Strecker, W.I. McAlexander, G. B. Partridge, and R. G. Hulet, Science 291, 2570 (2001).

[22] F. Schreck, L. Khaykovich, K. L. Corwin, G. Ferrari, T. Bourdel, J. Cubizolles, and C. Salomon, Phys. Rev. Lett. 88, 160401 (2002).

[23] Z. Hadzibabic, C. A. Stan, K. Dieckmann, S. Gupta, M. W. Zwierlein, A. Grlitz, and W. Ketterle, Phys. Rev. Lett. 88, 160401 (2002).

[24] G. Roati, F. Riboli, G. Modugno, and M. Inguscio, Phys. Rev. Lett. 89, 150403 (2002).

[25] P. A. Andreev, L. S. Kuz'menkov, Phys. Rev. A 78, 053624 (2008).

[26] L. S. Kuz'menkov, S. G. Maksimov, Theor. Math. Phys., 118, 287 (1999).

[27] P. A. Andreev and L. S. Kuz'menkov, arXiv:1201.2440

[28] P. A. Andreev and M. I. Trukhanova, Russian Physics Journal 53, 912 (2011).

[29] F. Haas, G. Manfredi, M. Feix, Phys. Rev. E 62, 2763 (2000).

[30] L. S. Kuz'menkov, S. G. Maksimov, and V. V. Fedoseev, Theor. Math. Fiz. 126, 136 (2001) [Theoretical and Mathematical Physics 126, 110 (2001)].

[31] M. Marklund and G. Brodin, Phys. Rev. Lett. 98, 025001 (2007).

[32] G. Brodin, M. Marclund, New J. Phys. 9, 277 (2007).

[33] G. Brodin, M. Marklund, J. Zamanian, B. Ericsson and P. L. Mana, Phys. Rev. Lett. 101, 245002 (2008).

[34] P. K. Shukla, B. Eliasson, Rev. Mod. Phys. 83, 885 (2011).

[35] F. A. Asenjo, V. Munoz, J. A. Valdivia, and S. M. Mahajan, Phys. Plasmas 18, 012107 (2011).

[36] P. A. Andreev, L. S. Kuz'menkov and M. I. Trukhanova, Phys. Rev. B 84, 245401 (2011).

[37] P. A. Andreev, arXiv: 1202.3398.

[38] P. A. Andreev, arXiv: 1201.0779.

[39] P. A. Andreev, arXiv: 1201.6553.

[40] D. A. Butts and D. S. Rokhsar, Phys. Rev. A 55, 4346 (1997).

[41] S. K. Adhikari, Phys. Rev. A 72, 053608 (2005).

[42] S. K. Adhikari, Journal of Physics B 38, 3607 (2005).

[43] S. K. Adhikari, New Journal of Physics 8, 258 (2006).

[44] Yu. V. Bludov, J. Santhanam, V. M. Kenkre, and V. V. Konotop, Phys. Rev. A 74, 043620 (2006).

[45] Matteo Rizzi and Adilet Imambekov, Phys. Rev. A 77, 023621 (2008).

[46] T. Maruyama and G. F. Bertsch, Phys. Rev. A 77, 063611 (2008).

[47] T. Karpiuk, M. Brewczyk and K. Rzewski, Phys. Rev. A 73, 053602 (2006). 
[48] A. M. Belemuk, V. N. Ryzhov, and S.-T. Chui, Phys. Rev. A 76, 013609 (2007).

[49] S. K. Adhikari, Phys. Rev. A 70, 043617 (2004).

[50] M. W. Zwierlein, J. R. Abo-Shaeer, A. Schirotzek, C. H. Schunck and W. Ketterle, Nature, 435, 1047 (2005).

[51] H. Washimi and T. Taniuti, Phys. Rev. Lett 17, 996 (1966).

[52] E. Infeld, G. Rowlands, Nonlinear waves, Solitons and Chaos, Cambridge University press, (2000).

[53] H.-Q. Cai, S.-R. Yang, J.-K. Xue, Commun. Theor. Phys. 55, 583 (2011).

[54] P. A. Andreev, L. S. Kuz'menkov, Russian Physics Journal
52, 912 (2009).

[55] P. A. Andreev, L. S. Kuzmenkov, arXiv:1105.5537

[56] N. N. Rosanov, A. G. Vladimirov, D. V. Skryabin, W. J. Firth, Phys. Lett. A. 293, 45 (2002).

[57] E. Braaten, H.-W. Hammer, and Shawn Hermans, Phys. Rev. A. 63, 063609 (2001).

[58] B. C. Kalita, R. Das, Plasma Phys. 5, 3588 (1998).

[59] Cheng Chin, et al., Rev. Mod. Phys. 82, 1225 (2010).

[60] I. Bloch, J. Dalibard, W. Zwerger, Rev. Mod. Phys. 80, 885 (2008). 